\title{
Chiral separation and twin-beam photonics
}

\author{
David S. Bradshaw and David L. Andrews* \\ School of Chemistry, University of East Anglia, Norwich Research Park, Norwich NR4 7TJ, U. K.
}

\begin{abstract}
It is well-known that, in a homogeneous fluid medium, most optical means that afford discrimination between molecules of opposite handedness are intrinsically weak effects. The reason is simple: the wide variety of origins for differential response commonly feature real or virtual electronic transitions that break a parity condition. Despite being electric dipole allowed, they manifest the chirality of the material in which they occur by breaking a selection rule that would otherwise preclude the simultaneous involvement of magnetic dipole or electric quadrupole forms of coupling. Although the latter are typically weaker than electric dipole effects by several orders of magnitude, it is the involvement of these weak forms of interaction that are responsible for chiral sensitivity. There have been a number of attempts to cleverly exploit novel optical configurations to enhance the relative magnitude - and hence potentially the efficiency - of chiral discrimination. The prospect of success in any such venture is enticing, because of the huge impact that such an advance might be expected to have in the health, food and medical sectors. Some of these proposals have utilized mirror reflection, and others surface plasmon coupling, or optical binding methods. Several recent works in the literature have drawn attention to a further possibility: the deployment of optical beam interference as a means to achieve chiral separations of sizeable extent. In this paper the underlying theory is fully developed to identify the true scope and limitations of such an approach.
\end{abstract}

Keywords: chirality, optical forces, single-molecule interactions, quantum theory, beam interference, chiral discrimination, optical manipulation, nanophotonics

\section{INTRODUCTION}

Chirality studies relating to optical forces that act on particles are receiving much current interest - for example, with respect to the Casimir force applied to chiral metamaterials ${ }^{1,2}$ or chiral objects, ${ }^{3-5}$ or induced chiral forces that arise due to the input of a laser beam. ${ }^{6-13}$ Research of the latter type involve optical discrimination for molecules of opposite handedness, which may lead to the separation of these enantiomers. If such works prove to be practicable at an industrial scale $^{14}$ a significant impact on the health, food and medical sectors is certainly possible. Our previous research ${ }^{8,15-18}$ worked $^{1}$ on the principle of optical trapping which, at the nanoscale, is described by forward-Rayleigh scattering of off-resonant input light; the resultant optical force is dependent on the position of the particle within the beam due to the non-uniform intensity profile of the beam. We established that a slightly different optical force arises when left-handed circularly polarized light is scattered by chiral molecules compared to right-handed light and, equivalently, when circular light of one type is scattered by a left-handed enantiomer compared to a right-handed one. A summary of these results is given in the next section. An explanation on the two different optical mechanisms that results in the center-of-mass motion of an uncharged, polarizable particle is then presented. The rest of section 3 gives an analysis on a two-beam system, based on a mechanism which is analogous to a four-wave mixing configuration, and involves forward-Rayleigh scattering of both off-resonant input beams. The article concludes with a brief discussion on the scope of the two beam set-up. 


\section{SEPERATION OF CHIRAL MOLECULES}

As described in the Introduction, optical trapping may be described by a forward-Rayleigh scattering mechanism. Equivalently it may also be represented by the dynamic (ac) Stark effect where an energy lowering of the electronic ground state occurs due to the alternating electric field of the input radiation. In quantum electrodynamical terms, both phenomena are defined in terms of the annihilation and creation of identical photons, i.e. instantaneous single absorption and single emission of photons with identical wavelengths within the transparent region of the particle. As in all chiral discrimination effects (which are always weak) the transition electric dipole contribution, denoted by E1, cannot be the origin of such effects on its own due to selection rule arguments. Since the mechanisms of interest here involves two photon-molecule interactions, it is the electric dipole-magnetic dipole (E1-M1) and electric dipole-electric quadrupole (E1-E2) contributions that are important rather the E1-E1 contributor, despite the latter usually being several orders of magnitude stronger. Typically, the Rayleigh scattering mechanism is sufficiently described by an electronic polarizability $\left(\alpha_{i j}\right)$ involving transition electric dipoles only, but the analogous E1-M1 $\left(G_{i j}\right)$ and E1-E2 $\left(A_{i j k}\right)$ polarizability tenors may be required instead in our case; the explicit forms of such tensors are given in ref. 8 .

The physical observable of optical trapping is a potential energy (or energy shift), $\Delta E$, which is dependent on position $\mathbf{r}$, may be defined in terms of identical initial and final states, denoted by $I$, and determined from second-order time-dependent perturbation theory, i.e.;

$$
\Delta E(\mathbf{r})=\sum_{R} \frac{\left\langle I\left|H_{\mathrm{int}}\right| R\right\rangle\left\langle R\left|H_{\mathrm{int}}\right| I\right\rangle}{E_{I}-E_{R}},
$$

where $R$ is an intermediate state, $E$ is the energy of the state denoted by the subscript and $H_{\text {int }}$ is the interaction Hamiltonian. When assuming a circularly polarized input beam of either specific handedness, the following expression is eventually found from equation $(1) ;^{8}$

$$
\Delta E^{(L \mid R)}(\mathbf{r})=-\left(\frac{I(\mathbf{r})}{2 \varepsilon_{0} c^{2}}\right)\left(c \bar{e}_{i}^{(L \mid R)} e_{j}^{(L \mid R)}\left(\alpha_{i j}+i k_{k}\left(A_{i j k}-A_{j i k}\right)\right)+\bar{e}_{i}^{(L \mid R)} G_{i j} b_{j}^{(L \mid R)}+\bar{b}_{i}^{(L \mid R)} \bar{G}_{j i} e_{j}^{(L \mid R)}\right)
$$

where $\mathbf{e}^{(L L R)} \equiv(1 / \sqrt{2})(\hat{\mathbf{i}} \pm \hat{\mathbf{j}})$ and $\mathbf{b}^{(L L R)} \equiv(1 / \sqrt{2})(\hat{\mathbf{j}}+i \hat{\mathbf{i}})$ are the circular polarization vectors of the electric and magnetic field, respectively $-\hat{\mathbf{i}}$ and $\hat{\mathbf{j}}$ are mutually orthogonal unit vectors perpendicular to the direction of light propagation and either left- or right-handed polarizations are denoted by the superscript - and $I(\mathbf{r})$ is the irradiance of the input beam. Assuming that the input beam is off-resonance with respect to the molecule's absorption bands, the terms involving the E1-E2 polarizability tenors may be omitted. This is justified since the energy shift is always a real quantity (a measurable), but the E1-E2 terms are imaginary since $A_{i j k}, A_{j i k}$ and $k_{k}$ (the wave-vector of the input beam) are all real quantities. The only case where these terms are non-zero is when the input beam is near-resonance, i.e. within the 'wings' of the resonance absorption band, since the E1-E2 polarizability tenors may then have an imaginary component - although, in such cases, a degree of absorption will occur and significantly weaken the desired effect. Similar arguments can be used to support the assertion that the final two terms of equations (2) cannot involve linearly polarized input light, since these terms will then be imaginary because the linear form of $\mathbf{e}$ and $\mathbf{b}$ are real quantities and $G_{i j}$ is purely imaginary; this correlates with the fact that circularly polarized light is more often used in chirality studies.

Determining the required expression for optical discrimination, i.e. the difference in potential energy for left-handed compared to right-handed circular polarization, requires application of the identity $\mathbf{b}^{(L / R)}=\mp i \mathbf{e}^{(L / R)}$ so that equation (2) becomes; 


$$
\Delta E^{(L)}(\mathbf{r})-\Delta E^{(R)}(\mathbf{r})=i\left(\frac{I(\mathbf{r})}{2 \varepsilon_{0} c^{2}}\right)\left(\bar{e}_{i}^{(L)} e_{j}^{(L)}+\bar{e}_{i}^{(R)} e_{j}^{(R)}\right)\left(G_{i j}-\bar{G}_{j i}\right)
$$

Here, the E1-E1 term cancels out (as expected) since identical results are found for either left- or right-handed circularly polarized input light; the remaining terms involve the $G_{i j}$ tensor only and, hence, the E1-M1 contribution is now solely considered. In greater depth, transition moments (whether electric or magnetic dipole, electric or magnetic quadrupole, etc.) exist for all molecules, it just depends on the electronic states they connect. A specific moment may be zero if the corresponding transition is symmetry forbidden, but all other cases will generally be non-zero. Most important in the present discussion is whether the $\alpha_{i j}, G_{i j}$ and $A_{i j k}$ tensors are non-zero. The former, E1-E1 polarizability tensor, is non-zero for all matter. On the other hand $G_{i j}$ (the E1-M1 tensor) is identically zero for some matter, such as centrosymmetric materials, because its structure involves products of E1 and M1 transition dipoles connecting the same pair of states (the ground and one other). These molecules may have allowed E1 transition dipoles and M1 transition dipoles too, but never connecting the same pair of electronic states. In contrast, E1 allowed transitions in all the symmetry point groups that support molecular chirality (the pure rotation groups) are always both E1 and M1 allowed, and also E1 and E2 allowed. In the present case, outside of selection rule arguments, the $A_{i j k}$ terms can be omitted since the observable $\Delta E$ has to be a real quantity and the $\alpha_{i j}$ terms are ignored since identical results are found for left- and right-handed light, as stated earlier.

It is presumed in equation (3) that chiral discrimination arises when a single enantiomer is irradiated with circularly polarized light and its response to left- and right-handed light differs. Significantly, identical outcomes occur for cases when a single circular polarization is applied to a left-handed chiral molecule relative to a right-handed one - this is the origin of enantiomer separation. The trapping force is determined using the expression $\mathbf{F}^{(\mathrm{LR})}(\mathbf{r})=-\nabla\left(U^{(\mathrm{LIR})}(\mathbf{r})\right)$, from which the differential chiral force is defined by $\Delta \mathbf{F}(\mathbf{r}) \equiv \mathbf{F}^{(\mathrm{L})}(\mathbf{r})-\mathbf{F}^{(\mathrm{R})}(\mathbf{r})$. Therefore, equation (3) corresponds to a non-zero trapping force for spatial separation of enantiomers in a fixed configuration; the corresponding results for freely rotating chiral molecules are found elsewhere. ${ }^{8,15,16}$

\section{ANALYSIS OF A TWO BEAM SYSTEM}

At this juncture it important to state that there are two forms of optical mechanism that result in the center-of-mass motion of a free, uncharged, polarizable particle. In the one of interest here, relating to the dynamic Stark shift, the particle's interaction with light produces intensity-dependent internal energy level shifts, introducing an effective potential energy surface wherever the light intensity varies with the particle position (most familiarly across the dimensions of a beam, as in optical tweezers). In this case the radiation field suffers no change in state, and the position-dependent particle energy can be evaluated as the expectation value of an interaction term which delivers the expression $\alpha \mathbf{E}^{2}$ (where $\boldsymbol{\alpha}$ is the polarizability tensor and $\mathbf{E}$ the optical electric field) in the electric dipole approximation. At a quantum level, this interaction involves photon annihilation and recreation into the same radiation mode - which can be cast as forwardRayleigh scattering in a Feynman diagrammatic sense (Figure 1). This is the origin of our scheme, and the mechanism is also the basis for numerous other works on optical forces. For example, it is consistent with optical lattice experiments in which the variation of internal energy with position serves as a potential energy surface and produces an optical dipole force.

Another mechanism describing center-of-mass motion comprises processes within which the radiation state suffers change, through a beam deflection and/or frequency shift, that is quantum mechanically (i.e. always in principle) observable. At the photon level this signifies the occurrence of scattering events in which there is an annihilation of photons in one mode, and the creation of photons in another mode with a different wave-vector. Accordingly the radiation field as a whole suffers a change in momentum; the corresponding momentum difference is imparted to the particle. The rate at which this process occurs determines the rate of change of momentum and hence the force, as in the familiar formula for radiation pressure. This is the basis for early research on optical trapping done by Ashkin, ${ }^{19}$ laser cooling techniques including optical molasses, ${ }^{20}$ and light pressure on free electrons. ${ }^{21}$ The difference in outcome, for the radiation, is such that no individual photon from a given optical input can manifest both types of mechanism, since the final radiation state 
cannot be both the same and different from the initial state. If a process with identifiably different radiation modes is involved - as, for example, in Doppler cooling - then the ensuing changes in particle motion are the result of a process that occurs at a well-defined rate. This involves the conversion of photons from one radiation mode into another. The crux is this: when the initial and final states differ in this way, the process is incoherent and, therefore, the physical observable depends on the square modulus of the quantum amplitude. ${ }^{22,23}$
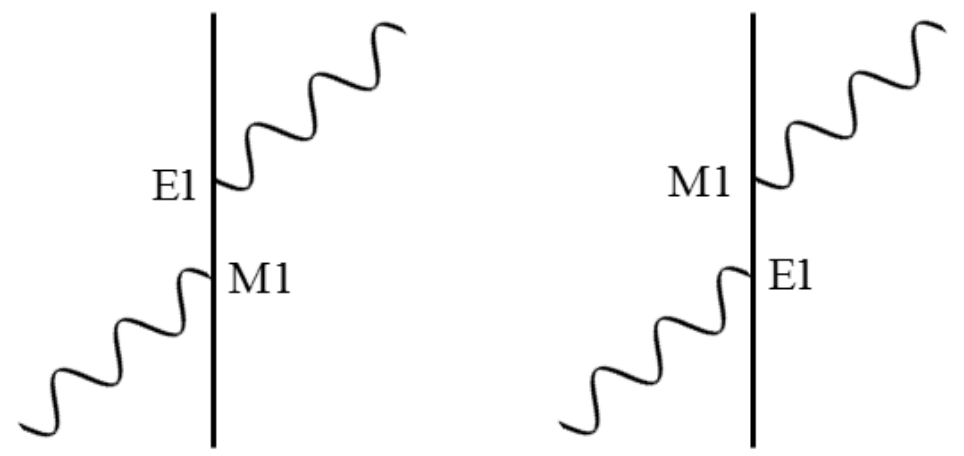

Figure 1. Feynman diagrams of forward-Rayleigh scattering mediated by a transition electric dipole (E1) and magnetic dipole (M1), namely the E1-M1 contribution. These diagrams correspond to chiral discrimination in the one beam set-up.

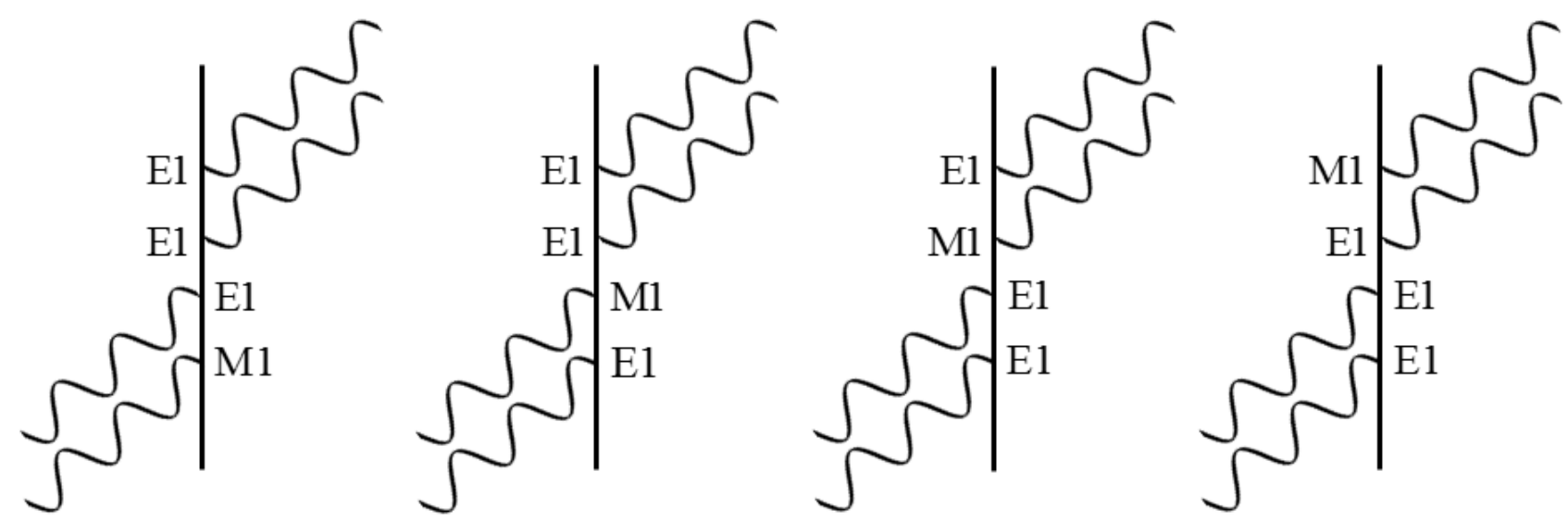

Figure 2. Feynman diagrams for forward-Rayleigh scattering of two beams mediated by three transition electric dipoles (E1) and a magnetic dipole (M1), where the four possible positions of M1 are shown. Diagrams relate to chiral discrimination in the two beam configuration.

Now returning to the first mechanism, where the initial and final states are required to be identical, the case of two irradiating beams is analyzed. Previously, for one input beam, the initial and final states correspond to a molecule $A$ in its ground state, 0 , and contains an occupant of a radiation mode; this may be denoted by $I \equiv\left|A^{0} ; 1\right\rangle$. For two irradiating beams these identical states will include an additional radiation mode, so that $I \equiv\left|A^{0} ; 1,1\right\rangle$ and two extra light-matter interactions are used; this is a higher-order (thus, weaker) process because of these additional interactions. In such a case, two photons are absorbed and two photons are emitted (Figure 2) - with both beams undergoing forward-Rayleigh scattering - and the potential energy is determined from perturbation theory, so that; 


$$
\begin{aligned}
\Delta E^{(L \mid R)}(\mathbf{r}) & =\left(\frac{I_{1}(\mathbf{r}) I_{2}(\mathbf{r})}{4 \varepsilon_{0}^{2} c^{3}}\right)\left(c \chi_{i j k l}^{E E E E}\left(\bar{e}_{1 i}^{(L \mid R)} e_{1 j}^{(L \mid R)} \bar{e}_{2 k}^{(L \mid R)} e_{2 l}^{(L \mid R)}\right)+\chi_{i j k l}^{E E E M}\left(\bar{e}_{1 i}^{(L \mid R)} e_{1 j}^{(L \mid R)} \bar{e}_{2 k}^{(L \mid R)} b_{2 l}^{(L \mid R)}\right)\right. \\
& \left.+\chi_{i j k l}^{E E M E}\left(\bar{e}_{1 i}^{(L \mid R)} e_{1 j}^{(L \mid R)} \bar{b}_{2 k}^{(L \mid R)} e_{2 l}^{(L \mid R)}\right)+\chi_{i j k l}^{E M E E}\left(\bar{e}_{1 i}^{(L \mid R)} b_{1 j}^{(L \mid R)} \bar{e}_{2 k}^{(L \mid R)} e_{2 l}^{(L \mid R)}\right)+\chi_{i j k l}^{M E E E}\left(\bar{b}_{1 i}^{(L \mid R)} e_{1 j}^{(L \mid R)} \bar{e}_{2 k}^{(L \mid R)} e_{2 l}^{(L \mid R)}\right)\right)
\end{aligned}
$$

which is similar to the result found in previous work for laser-induced optical rotation. ${ }^{24}$ In equation (4), $I_{1}$ and $I_{2}$ are the intensities of beam 1 and 2 respectively, the additional index on each polarization indicates the related beam, and the tensor $\chi_{i j k l}^{E E E E}$ is explicitly given by;

$$
\begin{aligned}
\chi_{i j k l}^{E E E E} & =\sum_{s}\left\{\frac{\bar{\alpha}_{k i}^{s 0} \alpha_{l j}^{s 0}}{E_{s 0}-\hbar \omega_{1}-\hbar \omega_{2}}+\frac{\bar{\alpha}_{l j}^{s 0} \alpha_{k i}^{s 0}}{E_{s 0}+\hbar \omega_{1}+\hbar \omega_{2}}+\frac{\bar{\alpha}_{l k}^{s 0} \alpha_{i j}^{s 0}}{E_{s 0}}\right. \\
& \left.+\frac{\bar{\alpha}_{j i}^{s 0} \alpha_{k l}^{s 0}}{E_{s 0}}+\frac{\bar{\alpha}_{l i}^{s 0} \alpha_{k j}^{s 0}}{E_{s 0}-\hbar \omega_{1}+\hbar \omega_{2}}+\frac{\bar{\alpha}_{k j}^{s 0} \alpha_{l i}^{s 0}}{E_{s 0}+\hbar \omega_{1}-\hbar \omega_{2}}\right\} \\
& \equiv R^{(1)}+R^{(2)}+R^{(3)}+R^{(4)}+R^{(5)}+R^{(6)},
\end{aligned}
$$

which corresponds to the situation where each of the four light-matter interactions involves transition electric dipole coupling. The other four tensors relate to one of the interactions mediated by a transition magnetic dipole in one of four possible positions, so that;

$$
\begin{array}{ll}
\chi_{i j k l}^{E E E M}=T^{(1)}+S^{(2)}+S^{(3)}+T^{(4)}+S^{(5)}+T^{(6)}, & \chi_{i j k l}^{E E M E}=S^{(1)}+T^{(2)}+S^{(3)}+T^{(4)}+T^{(5)}+S^{(6)}, \\
\chi_{i j k l}^{E M E E}=T^{(1)}+S^{(2)}+T^{(3)}+S^{(4)}+T^{(5)}+S^{(6)}, & \chi_{i j k l}^{M E E E}=S^{(1)}+T^{(2)}+T^{(3)}+S^{(4)}+S^{(5)}+T^{(6)},
\end{array}
$$

where $S^{(n)}$ is identical to $R^{(n)}$ except that the first $\boldsymbol{\alpha}^{\mathrm{s} 0}$ becomes a $\mathbf{G}^{\mathrm{s} 0}$ tensor, so (for example) $S^{(3)}=\bar{G}_{l k}^{s 0} \alpha_{i j}^{s 0} / E_{s 0}$, and similarly $T^{(n)}$ is the same as $R^{(n)}$ with the second $\boldsymbol{\alpha}^{\mathrm{s} 0}$ replaced by $\mathbf{G}^{\mathrm{s} 0}$. Determining an expression for optical discrimination for this case, again using the identity $\mathbf{b}^{(L \mid R)}=\mp i \mathbf{e}^{(L \mid R)}$, we find that the E1-E1 contributor will again cancel out and the following will then be found;

$$
\Delta E^{(L)}(\mathbf{r})-\Delta E^{(R)}(\mathbf{r})=i\left(\frac{I_{1}(\mathbf{r}) I_{2}(\mathbf{r})}{2 \varepsilon_{0}^{2} c^{3}}\right)\left(\bar{e}_{1 i}^{(L)} e_{1 j}^{(L)} \bar{e}_{2 k}^{(L)} e_{2 l}^{(L)}+\bar{e}_{1 i}^{(R)} e_{1 j}^{(R)} \bar{e}_{2 k}^{(R)} e_{2 l}^{(R)}\right)\left(\chi_{i j k l}^{E E E M}-\chi_{i j k l}^{E E M E}+\chi_{i j k l}^{E M E E}-\chi_{i j k l}^{M E E E}\right)
$$

A fourth-order rotational-average of this result, which physically means that the molecule is able to freely tumble within the laser beam (for example, within a fluid), will also give a non-zero outcome that is explicitly given by:

$$
\begin{aligned}
& \Delta E^{(L)}(\mathbf{r})-\Delta E^{(R)}(\mathbf{r})=i\left(\frac{I_{1}(\mathbf{r}) I_{2}(\mathbf{r})}{60 \varepsilon_{0}^{2} c^{3}}\right)\left\{8 \chi_{\mu \mu \nu v}^{E E E M}-8 \chi_{\mu \mu \nu v}^{E E M E}+8 \chi_{\mu \mu v v}^{E M E E}-8 \chi_{\mu \mu \nu v}^{M E E E}-2 \chi_{\mu \nu \mu \nu}^{E E E M}+2 \chi_{\mu \nu \mu \nu}^{E E M E}-2 \chi_{\mu \nu \mu \nu}^{E M E E}+2 \chi_{\mu \nu \mu \nu}^{M E E E}\right. \\
& -2 \chi_{\mu v v \mu}^{E E E M}+2 \chi_{\mu v v \mu}^{E E M E}-2 \chi_{\mu \nu v \mu}^{E M E E}+2 \chi_{\mu v \nu \mu}^{M E E E}+\left(\left(\overline{\mathbf{e}}_{1}^{(L)} \cdot \overline{\mathbf{e}}_{2}^{(L)}\right)\left(\mathbf{e}_{1}^{(L)} \cdot \mathbf{e}_{2}^{(L)}\right)+\left(\overline{\mathbf{e}}_{1}^{(R)} \cdot \overline{\mathbf{e}}_{2}^{(R)}\right)\left(\mathbf{e}_{1}^{(R)} \cdot \mathbf{e}_{2}^{(R)}\right)\right) \\
& \times\left(-\chi_{\mu \mu \nu v}^{E E E M}+\chi_{\mu \mu \nu v}^{E E M E}-\chi_{\mu \mu \nu v}^{E M E E}+\chi_{\mu \mu v v}^{M E E E}+4 \chi_{\mu \nu \mu v}^{E E E M}-4 \chi_{\mu \nu \mu v}^{E E M E}+4 \chi_{\mu \nu \mu v}^{E M E E}-4 \chi_{\mu \nu \mu v}^{M E E E}-\chi_{\mu v \nu \mu}^{E E E M}\right. \\
& \left.+\chi_{\mu \nu v \mu}^{E E M E}-\chi_{\mu \nu v \mu}^{E M E E}+\chi_{\mu \nu v \mu}^{M E E E}\right)+\left(\left(\overline{\mathbf{e}}_{1}^{(L)} \cdot \mathbf{e}_{2}^{(L)}\right)\left(\mathbf{e}_{1}^{(L)} \cdot \overline{\mathbf{e}}_{2}^{(L)}\right)+\left(\overline{\mathbf{e}}_{1}^{(R)} \cdot \mathbf{e}_{2}^{(R)}\right)\left(\mathbf{e}_{1}^{(R)} \cdot \overline{\mathbf{e}}_{2}^{(R)}\right)\right)\left(-\chi_{\mu \mu \nu v}^{E E E M}+\chi_{\mu \mu \nu v}^{E E M E}\right. \\
& \left.\left.-\chi_{\mu \mu \nu v}^{E M E E}+\chi_{\mu \mu v v}^{M E E E}-\chi_{\mu v \mu v}^{E E E M}+\chi_{\mu \nu \mu v}^{E E M E}-\chi_{\mu \nu \mu v}^{E M E E}+\chi_{\mu \nu \mu v}^{M E E E}+4 \chi_{\mu v \nu \mu}^{E E E M}-4 \chi_{\mu \nu v \mu}^{E E M E}+4 \chi_{\mu v v \mu}^{E M E E}-4 \chi_{\mu v v \mu}^{M E E E}\right)\right\} \text {, }
\end{aligned}
$$


where the Greek indices represent the molecule-fixed frame of reference. With a polarizability volume of $10^{-29} \mathrm{~m}^{3}$, the energy denominator of the polarizability as $10^{-19} \mathrm{~J}$, and an input beam intensity of $10^{11} \mathrm{~W} \mathrm{~cm}^{-2}$ we estimate that the two beam technique delivers a potential energy differential that is three orders of magnitude weaker than afforded by the single beam set-up.

Although optical discrimination is possible for a two beam configuration, the input beams needs to be circularly polarized rather than linearly polarized: this is justified since $\mathbf{e}, \mathbf{b}$ and $\boldsymbol{\alpha}$ are real quantities for linear light and $\mathbf{G}$ is imaginary, so the product of these tensors could not then give a potential energy that is real. For calculational convenience, the two input beams are assumed to be circularly polarized with the same handedness, but it is possible for the two beams to have different handedness as long as the corresponding handedness stays the same in the emission.

\section{DISCUSSION}

In the last few years chirality has become a hot topic, especially in physics and related subjects. The very latest, wide-ranging research discuss: the origin of homochirality in amino acids and sugars; ${ }^{25}$ the control of chirality in crystalline growth ${ }^{26}$ the separation of charge using chiral molecules; $;{ }^{27}$ the detection of enantiomers in a mixture through highharmonic generation, ${ }^{28,29}$ and chiral scattering as the source of orbital angular momentum transfer between an acoustic beam and matter. ${ }^{30}$ In this work, our novel mechanism for enantiomer separation is outlined and we can conclude that the additional complexities involved in a two-beam technique would not provide any advantages over a single beam set-up; the mechanism is higher-order than the one beam scenario and, thus, produces weaker effects and a differential chiral force that is three orders of magnitude smaller than the single beam case.

\section{ACKNOWLEDGMENTS}

The authors would like to thank the Leverhulme Trust and EPSRC for funding our research.

\section{REFERENCES}

[1] Zhao, R., Zhou, J., Koschny, T., Economou, E. N. and Soukoulis, C. M., "Repulsive Casimir force in chiral metamaterials," Phys. Rev. Lett. 103, 103602 (2009).

[2] Zhao, R., Koschny, T., Economou, E. N. and Soukoulis, C. M., "Comparison of chiral metamaterial designs for repulsive Casimir force,” Phys. Rev. B 81, 5 (2010).

[3] Butcher, D. T., Buhmann, S. Y. and Scheel, S., "Casimir-Polder forces between chiral objects," New J. Phys. 14, 113013 (2012).

[4] Hakami, J. and Zubairy, M. S., "Coherent control of Casimir force in a chiral medium,” J. Phys. B: At. Mol. Opt. Phys. 45, 205502 (2012).

[5] Hu, Y. H., Mao, C. X. and Hu, H., "Chiral force between a simple model of chiral molecules and a copper block induced by an axion," Chin. J. Phys. 50, 776-782 (2012).

[6] Canaguier-Durand, A., Hutchison, J. A., Genet, C. and Ebbesen, T. W., "Mechanical separation of chiral dipoles by chiral light," New J. Phys. 15, 123037 (2013).

[7] Shang, Q.-C., Wu, Z.-S., Qu, T., Li, Z.-J., Bai, L. and Gong, L., “Analysis of the radiation force and torque exerted on a chiral sphere by a Gaussian beam," Opt. Express 21, 8677-8688 (2013).

[8] Bradshaw, D. S. and Andrews, D. L., "Chiral discrimination in optical trapping and manipulation," New J. Phys. 16, 103021 (2014).

[9] Cameron, R. P., Barnett, S. M. and Yao, A. M., "Discriminatory optical force for chiral molecules," New J. Phys. 16, 013020 (2014).

[10] Cameron, R. P., Yao, A. M. and Barnett, S. M., "Diffraction gratings for chiral molecules and their applications," J. Phys. Chem. A 118, 3472-3478 (2014). 
[11] Canaguier-Durand, A. and Genet, C., "Chiral near fields generated from plasmonic optical lattices,” Phys. Rev. A 90, $023842(2014)$.

[12] Chen, H., Jiang, Y., Wang, N., Lu, W., Liu, S. and Lin, Z., "Lateral optical force on paired chiral nanoparticles in linearly polarized plane waves," Opt. Lett. 40, 5530-5533 (2015).

[13] Alizadeh, M. H. and Reinhard, B. M., "Transverse chiral optical forces by chiral surface plasmon polaritons," ACS Photonics, (in press).

[14] Ahuja, S., [Chiral Separation Methods for Pharmaceutical and Biotechnological Products], Wiley, New York (2011).

[15] Bradshaw, D. S. and Andrews, D. L., "Laser optical separation of chiral molecules,” Opt. Lett. 40, 677-680 (2015).

[16] Bradshaw, D. S. and Andrews, D. L., "Electromagnetic trapping of chiral molecules: Orientational effects of the irradiating beam," J. Opt. Soc. Am. B 32, B25-B31 (2015).

[17] Bradshaw, D. S., Forbes, K. A., Leeder, J. M. and Andrews, D. L., "Chirality in optical trapping and optical binding," Photonics 2, 483-497 (2015).

[18] Bradshaw, D. S., Leeder, J. M., Coles, M. M. and Andrews, D. L., "Signatures of material and optical chirality: Origins and measures," Chem. Phys. Lett. 626, 106-110 (2015).

[19] Ashkin, A., "Acceleration and trapping of particles by radiation pressure," Phys. Rev. Lett. 24, 156-159 (1970).

[20] Lett, P. D., Phillips, W. D., Rolston, S. L., Tanner, C. E., Watts, R. N. and Westbrook, C. I., "Optical molasses,” J. Opt. Soc. Am. B 6, 2084-2107 (1989).

[21] Kaplan, A. E., "Fully QED/relativistic theory of light pressure on free electrons by isotropic radiation," J. Phys. B: At. Mol. Opt. Phys. 48, 165001 (2015).

[22] Marcuse, D., [Engineering Quantum Electrodynamics], Harcourt, Brace \& World, San Diego (1970).

[23] Andrews, D. L. and Allcock, P., [Optical Harmonics in Molecular Systems], Wiley-VCH, Weinheim (2002).

[24] Thirunamachandran, T., "Theory of laser-induced optical activity," Proc. R. Soc. A 365, 327-343 (1979).

[25] Jafarpour, F., Biancalani, T. and Goldenfeld, N., "Noise-induced mechanism for biological homochirality of early life self-replicators," Phys. Rev. Lett. 115, 158101 (2015).

[26] Damasceno, P. F., Karas, A. S., Schultz, B. A., Engel, M. and Glotzer, S. C., "Controlling chirality of entropic crystals," Phys. Rev. Lett. 115, 158303 (2015).

[27] Peer, N., Dujovne, I., Yochelis, S. and Paltiel, Y., "Nanoscale charge separation using chiral molecules," ACS Photonics, (in press).

[28] Cireasa, R., Boguslavskiy, A. E., Pons, B., Wong, M. C. H., Descamps, D., Petit, S., Ruf, H., Thire, N., Ferre, A., Suarez, J., Higuet, J., Schmidt, B. E., Alharbi, A. F., Legare, F., Blanchet, V., Fabre, B., Patchkovskii, S., Smirnova, O., Mairesse, Y. and Bhardwaj, V. R., "Probing molecular chirality on a sub-femtosecond timescale," Nature Physics 11, 654-658 (2015).

[29] Smirnova, O., Mairesse, Y. and Patchkovskii, S., "Opportunities for chiral discrimination using high harmonic generation in tailored laser fields," J. Phys. B: At. Mol. Opt. Phys. 48, 234005 (2015).

[30] Wunenburger, R., Lozano, J. I. V. and Brasselet, E., “Acoustic orbital angular momentum transfer to matter by chiral scattering," New J. Phys. 17, 103022 (2015).

*david.andrews@physics.org 